\title{
Exploration on the construction of experimental teaching demonstration center of economics and management
}

\author{
Rongrong Lin, Heqin Zhao \\ International Business Faculty, Beijing Normal University, Zhuhai, 519000, China. \\ Email: rrlin@bnuz.edu.cn
}

Keywords: Experimental center; Internationalization; Experimental system; Innovation.

\begin{abstract}
The trend of internationalization of college education has put forward new requirements for the construction of experimental center of economics and management. Taking the construction of the experimental teaching demonstration center of economics and management in Beijing Normal University Zhuhai as an example. To combine with their own characteristics, focus on features of internationalization, grand business, and diversity. To train extroverted, applied and compound international talents, develop students' ability of international applications and vision, promote their international competitiveness.
\end{abstract}

\section{Introduction}

Beijing Normal University, Zhuhai (hereinafter referred to as the "BNUZ") is a full-time college of undergraduate level education, it is an important part of the construction of "double top-class" university by Beijing Normal University [1]. The experimental teaching demonstration center of economics and management (hereinafter referred to as the "center") was set as the construction of the quality engineering project of BNUZ in 2012. In 2015, the center was set as the construction of the quality engineering project of Guangdong province. The center has a teachers' team with high level of education, strong business ability and patience. It has invested over 30,000,000 yuan, more than 3,000 square meters buildings, 1,000 parts of all kinds of experimental equipment instruments, 40 sets of professional experimental teaching software. It has set up 40 experimental courses and nearly 500 experimental contents, of which $70 \%$ were comprehensive and innovative experiments. The renewal rate of experimental content is over 5\% every year. The center has 9 laboratories, named teaching simulation lab, professional training lab, digital voice lab, finance lab, accounting lab, business administration lab, e-commerce lab, international trade lab and computer lab. Every year, about 6,000 students come to the center to do experiments, majored in finance, accounting, business administration, e-commerce, international economy and trade, etc.

\section{Develop goals of the center}

The center takes the features of internationalization, big business and diversity. It aims to create the innovation of contemporary commercial culture and to develop extroverted, applied and compound international talents, who has strong professional knowledge, high English level, strong operational ability, knowledge of frontier disciplines, international vision and modern concept.

The center always adheres to the idea of taking students as the base and taking ability training as the core. It emphasizes the innovation of talents training, strengthen practice means, reform teaching content and method, increase the construction of training base and experimental practice teaching sharing platform, highlight education of high quality and scientific and technological culture innovation. The development path is structure optimize, features enhance, innovation emphasis and quality improve. According to the standard of national experimental teaching demonstration center [2], the center should be built as a cross subject, cross specialty experimental teaching platform, collaborative platform of innovation and international cooperate platform, covering the experimental teaching and the practice teaching of social innovation and entrepreneurship of the whole school. 


\section{Construction of experimental system}

The innovative experimental system is not simply to divide the experimental courses, contents and projects in accordance with the basic courses, professional courses and graduated design. According to the basic rules of experimental teaching, and for the purpose of the training of creative talents, the whole part of the experimental teaching is systematically designed. Figure 1 is the schematic diagram of the experimental system. A complete experimental system includes four parts: the teaching system (target), the teachers' team (the main body), the supervision and encouragement (means) and the management mechanism (guarantee), they are interrelated and jointly promote the development of the center.

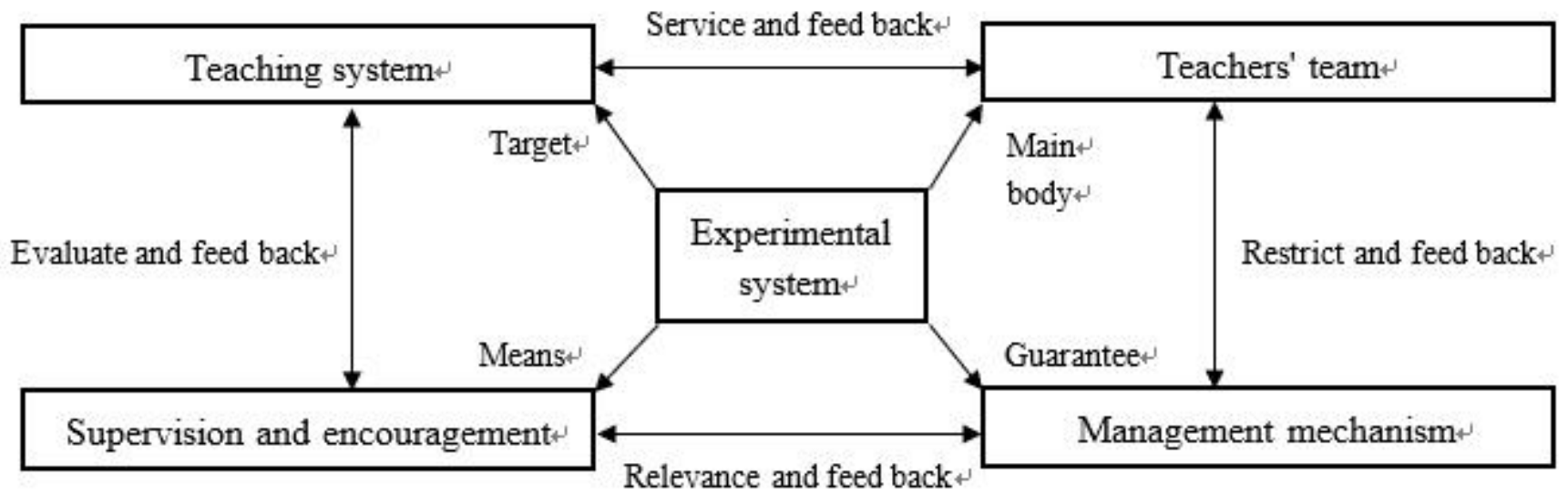

Figure 1 The schematic diagram of the experimental system

\subsection{Teaching system}

The internationalized talents must have the following capabilities: the ability of professional knowledge and basic skills, the ability of using information technology, the ability to collect and analyze data, the ability to use foreign language, the coordinate and communicate ability of international cooperation, and the ability of teamwork, and so on. Based on the requirements of these capabilities, bold innovation and reform have been carried out in the construction of the experimental teaching system.

\subsubsection{Scientific and rational target of talent training}

Based on reality and focusing on the future, according to the outline of the national medium and long term educational reform and development plan [3], combining with the comprehensive level of the school, the center establishes the training target of scientific thinking, innovative spirit and internationalized accomplishment, serving the national strategic requirements and regional economic development.

\subsubsection{Optimization of experimental course system}

The center actively promotes the optimization and innovation of the experimental curriculum system, integrates the experimental courses of various specialties, reforms the experimental teaching mode and optimizes the experimental scheme, and divides the experimental types into three types: basic experiment, comprehensive experiment and innovative experiment.

The basic experiment course is mainly to carry out experiment of verification and simulation, the experiment content and method are relatively simple. The students, under the guidance of the teacher, complete the experiment process according to the established methods and conditions. This kind of experiment course is usually arranged in grade one or two, and the experiment course is supplemented as the basic theory course to deepen the students' understanding and mastery of theoretical knowledge. In recent years, with the continuous optimization of experimental courses, the proportion of basic experimental courses has declined year by year, from the original $50 \%$ to the current 30\%. The basic experiment courses include computer basis, electronic business technology basis, business statistics analysis, securities technology analysis, international import and export 
trade simulation, etc.

On the basis of certain professional basic knowledge, the comprehensive experiment course arranges the experiment of design and inquiry. The experiment content, method and means synthetically inspects the students' experimental ability. This kind of class is generally arranged in grade two or three. Students have high autonomy in the experiment course. They need to explore, investigate, visit, inspect, operate and service according to the knowledge they have learned, and improve the ability of comprehensive practice. The comprehensive experimental course accounts for $50 \%$ of the experimental courses, including ERP sand table business, e-commerce website design, business negotiation, human resource studio, etc.

The purpose of the study of innovative experiments is to strengthen the students' research and study ability through experiments, develop students' potential, cultivate creative thinking, and achieve the organic combination of teaching and scientific research. The center encourages students to carry out innovative experiments, arrange guidance teachers, research sites, equipment and support for innovative experimental projects, and reward research results published or elected to international academic conferences in important publications, and form a good encouraged mechanism. Under the guidance of the experimental teachers, every year, students publish academic papers at international conferences and receive state-level university students' entrepreneurial and innovated projects.

\subsubsection{Innovation of experimental teaching content}

The center insists on keeping pace with the times, focusing on the needs of social development, and constantly increasing the input and teaching of new concepts and technologies. Build a bilingual training platform of international business, virtual simulate [4] international business environment and business activities, complete the core curriculum learning, professional practice and cross professional simulation training, help students to build comprehensive ability of facing the actual problems and solve the problems. Carry out the construction of cloud data and Massive Open Online Courses (MOOCs), make full use of the new technology and new means of "Internet +" [5] education and wisdom education, make the experimental teaching ubiquitous, educational resources integrated, in order to cultivate innovative talents. Organic integration of the resources of schools, government and enterprises, gradually form a comprehensive entrepreneurship training mode. Entrepreneurial education is carried out through entrepreneurial training, competition, innovated program, and entrepreneurial real war education is carried out in hackerspace, training base and industrial incubator. The center cultivates students' innovative consciousness, entrepreneurial quality and practical ability step by step through stratified education.

\subsection{Teachers' team}

The teachers' team has an important impact on laboratory construction and talents training. At present, there are 31 experimental teachers in the center, including 25 full-time experimental teachers, 6 part-time workers (all are experts with professional background), and 20 with senior professional titles and 15 doctorates. The center regularly organizes teachers in a variety of academic seminars and exchanges, such as organizing and participating in the ISO and TQM international academic conferences, the symposium of deans of foreign business schools, and other professional and technical training (ERP, SAP, SPSS, EVIEWS, etc.). The center sends teachers to cooperated universities (the University of Leicester, the Saint Mary's University, etc.) to carry out short-term training or exchange as a visiting scholar, and organize teachers to visit enterprises (Alibaba, UF, GTA, etc.). Efforts through multiple channels should be made to improve the professional quality, teaching level and scientific research ability of the teachers [6].

\subsection{Management mechanism}

A good experimental system cannot be separated from a scientific and reasonable management mechanism. The center is directly managed by the International Business Faculty (IBF) of BNUZ. The experimental teachers, technicians and managers of the center are distributed by IBF. It weakens the traditional boundaries between the various specialized experimental courses, breaks the barriers between the subjects, emphasizes the integration of the subjects and the sharing of resources, and is 
beneficial to experimental teaching. In addition, according to the relevant regulations of the school, the center set up a series of policies and measures, rules and regulations, experimental process, and so on, to ensure the smooth progress of experimental teaching and the safety and stability of laboratory construction.

\subsection{Supervision and encouragement}

The two-level supervision system [7] of the school and the center is set up. The center takes the form of whole course monitoring, random attendance, spot check of teaching plan and experiment report, examination, and experimental informal discussion to test and evaluate the experimental teaching, which can effectively change the traditional concept of light experiment teaching and stop the problems such as being late, leaving early, shortening class hours, inconsistent contents, and adjusting classes privately.

In order to improve the status and treatment of the experimental teachers, the experimental teaching and theoretical teaching are calculated on the same amount of work, and teachers are encouraged to arrange and add new experimental classes according to their course, and teachers are encouraged to use the new teaching technologies, such as MOOCs and mobile terminal, to carry out the experiment course. The experimental teachers can use the notebook computer and other equipment first. Take precedence over the excellent teaching award to the teachers of the experimental class, improve the performance reward. Give the teachers who lead students to participate in the national or provincial experiment competition and win the prize, priority in the title and scientific research project evaluation.

\section{Features of the center}

\subsection{International cooperative education}

In order to meet the needs of international cooperative education, the center has built a remote conference system which can communicate with foreign cooperated universities in real time, achieving the "zero distance" between students and foreign experts. The construction of the big screen display system, the horse running light system, the stock trading hall, make it possible for students to understand the foreign exchange, futures, securities and other information in real time and carry out operation. The center has purchased a number of English or bilingual software, set up courses that are required in syllabus of foreign cooperated universities, such as SPSS business statistics application, the application of Excel in finance, NVIVO qualitative analysis and so on. The center also works with companies to build a bilingual and interdisciplinary integrated training platform for international business, enabling students to emulate the business environment and activities of different cultural backgrounds in the laboratory, and to improve the international competitiveness in an all-round way.

\subsection{Multidisciplinary intersecting}

The experiments are set to be multidisciplinary and multi professional intersecting [8], and the services and resources are highly integrated and shared, so as to meet the urgent need of comprehensive and internationalized talents of the society. At present, in addition to meeting the requirements of the experimental courses stipulated in the undergraduate syllabus of IBF, the teaching arrangements and resources for the practical courses are provided to the College of Applied Mathematics, the College of Franchise, and the College of Real Estate, etc.

\subsection{The industrial-academic-research cooperation}

The center works cooperate with famous research institutions and enterprises such as IBM, UFIDA, ZS, LD in teaching software development, experiment teaching and professional practice. For a long time, IBM provides free teacher training, teaching software, lectures, and course tour, cooperates in global professional certification training and textual research in many courses such as DB2, LOTUS, LINUX. IBM set up SPSS analysis and application courses for students free of charge 
in short semester. The center put forward revise opinions in the development of the Commercial Bank Business Simulation Software of the ZS Company. In cooperation with the UFIDA company, the center carries out various vocational qualification certificate training, send teachers to take part in training and various learning experience activities such as ERP sand table and electronic sand table, and organize students to participate in various competitions, such as the national ERP electronic sand table competition, accounting skills competition, and quantitative investment competition. An e-business studio is set up in the school with the LD Company, students would be able to participate in e-commerce business activities such as online sales.

\subsection{Resource sharing}

The center adheres to the sharing of the experimental teaching facilities and equipment. Most of the software are B/S structures and are opened all day to all students. Teaching courseware and experimental instruction can be downloaded through the center's website. The experimental materials, sand table, notebook computer and other hardware equipment can be borrowed free of charge. All laboratories are open after class and students can study independently or carry out experimental research.

\subsection{Serving local economic construction}

In the past three years, the center's teachers have applied for more than 20 provincial and municipal vertical scientific research projects and completed more than 30 horizontal scientific research projects of the city, district and listed companies, providing decision-making and consulting services for enterprises and municipal and district governments, which are well received by the government and enterprises, making great contribution to the local economic construction.

\section{Conclusions}

The construction of the center is a complex and long-term systematic project. The center has made the following efforts: first setting up the international teaching system, second developing the teachers' team of high professional quality, third formulating comprehensive and meticulous rules and regulations, and fourth establishing efficient and strict supervision and encouraged mechanism. At present, a certain achievement has been made in following the trend of internationalization, adopting new technology, doing scientific research, carrying out the cooperation of government, school and enterprise, improving and perfecting the experimental system, it has laid a solid foundation for training talents who meet the fierce competition in the world.

\section{Acknowledgements}

This work was financially supported by the construction of the experimental teaching demonstration center of economics and management of the quality engineering project of Guangdong province (2015306); Program for research development of Beijing Normal University, Zhuhai.

\section{References}

[1] Ning Kang, Qi-long Zhang, Hui-bin Su. Tsinghua Journal of Education, 2016, 37(5):11-19 (in Chinese).

[2] Li-gui Wang. Research and Exploration in Laboratory, 2011, 30(2):98-100 (in Chinese).

[3] Li Cui, Li Liang. Education Exploration, 2012(12):97-98 (in Chinese).

[4] Xin-mei Guo, Cheng-xin Fang. Experimental Technology and Management, 2014, 31(11):8-12 (in Chinese).

[5] Rong-rong Lin, He-qin Zhao. Foreign Economic Relations \& Trade, 2017(8):73-74+108 (in Chinese). 
[6] Hong Li, Xian-chun Guo, Dan Li. Modern Education Management, 2015(10):58-62 (in Chinese).

[7] Qing-wei Dong, Yong-zhen Zhang, Ke-qin Tong, Jing-han You, Rui Fan. Experimental Technology and Management, 2013, 30(7):147-149 (in Chinese).

[8] Hua Liu, Rong Zhu, Chun Sun, Rui-ting Li, Yu Li. Laboratory Science, 2017,20(1):162-164 (in Chinese). 